\title{
A Convex Optimization Approach to Cancer Treatment to Address Tumor Heterogeneity and Imperfect Drug Penetration in Physiological Compartments
}

\author{
Giulia Giordano $^{a}$, Anders Rantzer ${ }^{a}$ and Vanessa D. Jonsson ${ }^{b}$
}

\begin{abstract}
The clinical success of targeted cancer therapies is limited by the emergence of drug resistance often due to pre-existing tumor genetic heterogeneity and acquired, therapyinduced resistance. Targeted therapies have varied success in addressing metastatic disease, due to their ability to penetrate certain physiological compartments. This paper considers an evolutionary cancer model that incorporates tumor cell growth, mutation and compartmental migration and leverages recent results on the optimal control of monotone and convex systems to synthesize switching treatment strategies where a single drug or a predetermined combination of drugs is used at a given time. The need for switching is motivated by clinical considerations such as the limited effectiveness of any single targeted therapy against multiple resistance mechanisms arising in a single patient and the inability to design drug combinations at effective doses due to toxicity constraints. An optimal and clinically feasible switching therapy is obtained as the solution of a convex optimization problem that exploits the diagonally-dominant structure of the model. We demonstrate that this method yields an effective strategy in mitigating disease evolution in the presence of imperfect drug penetration in two compartments on an experimentally identified model of anaplastic lymphoma kinase (ALK)-rearranged lung carcinoma.
\end{abstract}

\section{INTRODUCTION AND MOTIVATION}

Targeted therapies are effective for the treatment of certain oncogene-driven tumors [1]; however, clinical response is transient and often within months of treatment initiation, resistance emerges [2], [3], [4]. These small molecule inhibitors and monoclonal antibodies commonly exploit particular genetic addictions and vulnerabilities, and establish an environment in which the occurrence of mildly drug resistant cells can develop an evolutionary advantage over those for which the therapy is targeted [5], [6]. Clonal expansion of these evolutionary advantageous cells is exacerbated by the considerable genetic intratumoral heterogeneity often already present in treatment-naive patients [7].

There is also evidence that treatment with certain targeted therapies has poorer prognosis for patients with metastatic disease. Often, imperfect penetration of certain physiological compartments can lead to sub-therapeutic drug levels that dramatically increase the chance of overall treatment failure [8]. Some inhibitors have better prognosis for patients with

a Department of Automatic Control LTH and LCCC Linnaeus Center, Lund University, Box 118, 22100 Lund, Sweden. \{giulia.giordano, rantzer\}econtrol.lth.se

$b$ Department of Hematology \& Hematopoietic Cell Transplantation, Beckman Research Institute at City of Hope National Medical Center, Duarte, CA 91010. vjonssondeoh.org. metastatic disease, due to an increased ability to penetrate certain physiological compartments, whereas others are typically not found in high enough concentrations to have a clinically relevant effect [9], [10]. The lack of drug permeability in compartments such as the central nervous system (CNS) makes brain metastases notoriously difficult to treat from a pharmacological perspective. In addition to potential spatial heterogeneity in drug concentrations, constant treatment strategies with small molecule inhibitors fail to control progression of heterogeneous tumors [2], [3], [4]. As an alternative, drug pulsing [11] or treatment switching strategies have been proposed and shown to better control tumor growth [12].

The challenge of designing treatment protocols that prevent or delay resistance and progression in cancer has been explored in several control-theoretic settings. Results in optimal and receding horizon control [13], [14] and gain scheduling [15] have been applied to synthesize treatment protocols that are robust to parameter uncertainty. In [16], static multi-objective optimization is used on experimentally derived dose response data to solve the combination therapy problem for different initial tumor populations, when the drugs under consideration have additive, linear effects on cell viability. These predicted combination treatments have been validated experimentally in a murine lymphoma model for different tumor initial conditions [17].

In the context of HIV treatment design, switching strategies that minimize the total viral load within a finite horizon, thus mitigating viral escape in the presence of mutations, can be designed by solving an optimal control problem [18] whose cost functional is convex [19]. To prevent the emergence of resistant mutants, infinitely fast switching [20] may be needed. However, this strategy is clinically unfeasible and to address this, a dwell-time constraint on the switching rule is included [21] to ensure that a minimum amount of time elapses between drug switching. A model-predictivecontrol approach has been proposed to solve the optimal control problem over a short receding horizon [22].

Here, we introduce a multi-compartment population model that encodes the evolutionary dynamics of heterogeneous tumor cells undergoing replication, genetic mutation, migration (to compartments) and drug response, accounting for imperfect drug penetration in physiological compartments (Section II). Based on recent results in optimal control of monotone and convex-monotone systems [19], [23], [24], in Section III we propose an algorithm to design optimal 
and clinically feasible switching treatment strategies that minimize tumor progression when drug pharmacodynamics are subject to maximum dosage constraints, the drug dose is piecewise-constant in time, and only one drug is used at a given time. We consider linear drug dose responses and exploit the biological fact that growth phenomena are dominant with respect to mutation and migration to formulate a convex programming problem. Indeed, when the state matrix associated with the model is practically diagonal, the tumor reduction depends exclusively on the overall duration of the treatment with each drug, and not on the moment when each drug is used: this yields both the advantages of convex optimization and more freedom in therapy scheduling. Section IV illustrates the proposed strategy for synthesizing switching treatments over a finite horizon using a model of metastatic ALK rearranged lung adenocarcinoma: switching therapies offer a good control of the overall disease progression. Section V ends with concluding remarks and directions for future work.

Notation. $\mathbb{R}_{+}$denotes the set of nonnegative real numbers, $\mathbf{1}_{n}$ the ones vector of dimension $n$. The inequality $X>0$ $(X \geq 0)$ means that all elements of the matrix or vector $X$ are positive (nonnegative). Matrix $A \in \mathbb{R}^{n \times n}$ is Metzler if all its off-diagonal elements are nonnegative.

\section{Multi-Compartment Evolutionary Model}

We include drug dose response [25] and compartment dynamics in the well-known quasi-species evolutionary model [26], [27] and propose a multi-compartment evolutionary model that describes growth, mutation, metastasis and selection by drugs of a heterogeneous tumor cell population. In the presence of $m$ mutant cell lines, of migrations among $p$ compartments and of $d$ different drugs (or suitable drug combinations), the concentration $x_{i}^{k} \in \mathbb{R}_{+}$of mutant species $i$ in compartment $k$ evolves as

$$
\begin{aligned}
\dot{x}_{i}^{k} & =r_{i}^{k} q_{i i, k} x_{i}^{k}+\sum_{\substack{j=1 \\
j \neq i \\
j}}^{m} r_{i}^{k} q_{i j, k} x_{j}^{k} \\
& +\sum_{c=1}^{p} \mu_{k c, i} x_{i}^{c}-\sum_{\substack{c=1 \\
c \neq k}}^{p} \mu_{c k, i} x_{i}^{k}-\Psi_{i}^{k}(\ell) x_{i}^{k},
\end{aligned}
$$

where $r_{i}^{k}$ is the growth rate of mutant $i$ in compartment $k, q_{i j, k}$ is the probability that mutant $j$ mutates to mutant $i$ in compartment $k$ (hence, $q_{i i, k}$ is the probability of no mutation occurring), $\mu_{c k, i}$ is the migration rate of mutant $i$ from compartment $k$ to compartment $c$. Growth rates, as well as mutation and migration dynamics (depicted in Fig. 1), are assumed to be independent of drug effects. The vector of drug concentrations $\ell=\left(\ell_{s}\right) \in \mathbb{R}_{+}^{d}$ is assumed to have piecewise-constant components $\ell_{s}$, while the function $\Psi_{i}^{k}(\ell)$ represents the overall drug dose response in compartment $k$ with respect to mutant $i$.

When $\ell_{s}=0 \forall s \in\{1, \ldots, d\}$, the dynamics in Eqn. (1) represent tumor growth and metastasis in the absence of therapy and are unstable. The contribution of each individual

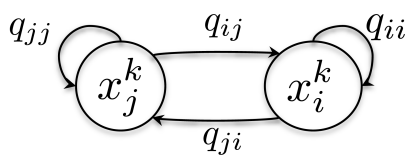

(A)

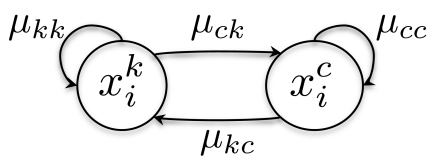

(B)
Fig. 1. Pairwise mutation dynamics (A) and pairwise compartment migration dynamics (B), whose overall effect is included in Eqn. (1).

drug to function $\Psi_{i}^{k}(\ell)$ can be modeled as follows: the bound cell receptors $\rho$ are quantified as a function of the ligand (i.e., drug) concentration $\ell_{s}$ according to the drug-receptor binding reaction $\ell_{s}+\rho \stackrel{K_{A}}{\longrightarrow} \ell_{s} \cdot \rho$, corresponding to the Hill function

$$
\psi_{s, i}^{k}\left(\ell_{s}\right)=\frac{\left(\ell_{s}\right)^{n_{s}}}{\left(\ell_{s}\right)^{n_{s}}+K_{s}^{n_{s}}},
$$

where $\psi_{s, i}^{k} \in[0,1]$ is the fraction of bound receptors relative to mutant $i$, compartment $k$ and drug $s, \ell_{s} \in \mathbb{R}_{+}$is the ligand (drug) concentration, $K_{s}=\frac{1}{K_{A}} \in \mathbb{R}_{+}$is the dissociation constant of the binding reaction, and the Hill coefficient $n_{k} \in$ $\mathbb{R}_{+}$represents the cooperativity index (i.e., the degree to which binding of a ligand molecule modulates the probability of another ligand molecule binding). For small enough drug amounts, far from saturation, the drug effects can to a good approximation be assumed linear:

$$
\psi_{s, i}^{k}\left(\ell_{s}\right)=K_{s, i} \ell_{s}, \quad K_{s, i}>0 .
$$

For an additive drug model, in which drugs do not interact competitively, neither synergistically nor antagonistically, the total effect of independently acting drugs $\ell_{1}, \ldots, \ell_{d}$ on mutant $i$ in compartment $k$ could be described as

$$
\Psi_{i}^{k}(\ell)=\sum_{s=1}^{d} \psi_{s, i}^{k}\left(\ell_{s}\right)
$$

Additive drug models are relevant for a large class of cancer drugs [28] but are not generalizable to all targeted therapies[29]. Drug combinations often include therapies that selectively target different genes on the same or a related signaling pathway. Their combined effect can invoke changes to the underlying signaling network dynamics and can result in non-additive effects [29]. In our formulation, we consider clinically relevant drug doses and assume that combination drug effects are known at these doses. We then synthesize piecewise-constant switching treatment strategies such that only one drug (or a suitable drug combination with a known combined effect) is selected at any given time, and used at a corresponding maximum tolerated dose (MTD).

To further emphasize the inherent feedback structure that arises from drug effect on the growth, mutation and migration of tumor cell populations, we rewrite the overall model as

$$
\dot{x}(t)=[A-\Psi(\ell(t))] x(t),
$$

where $x=\left(x_{i}^{k}\right)$ is the stacked vector of tumor cell populations, having size $n=m p$, while matrices $A$ and $\Psi$ are chosen such that the dynamics (5) are consistent with (1): $A \in \mathbb{R}^{n \times n}$ is an unstable Metzler matrix and the drug effects are described by the diagonal matrix $\Psi(\ell) \in \mathbb{R}^{n \times n}$, whose 
diagonal entries are increasing functions of $\ell$.

Remark 1: Due to the biological quantities involved, the off-diagonal entries of matrix $A$ are several orders of magnitude smaller than its diagonal entries.

Since the drug doses we consider are not greater than the MTD, the diagonal entries of $\Psi(\ell)$ can be well approximated as linear functions of $\ell$. Hence, the model becomes

$$
\dot{x}(t)=\left[A-\sum_{s=1}^{d} D_{s} \ell_{s}(t)\right] x(t),
$$

where $D_{s} \in \mathbb{R}^{n \times n}$ is a positive diagonal matrix expressing the effect of drug $s$.

\section{Synthesis OF Optimal SWitching TREATMEnTS}

Suitable treatment strategies to address intratumor heterogeneity, imperfect drug penetration and resulting resistance can be designed by solving an optimal control problem. For all drugs, we normalize the maximum tolerated dose (MTD) to $\ell_{s}=1$. Then, our goal is to design an optimal switching therapy that minimizes the final tumor size $\mathbf{1}_{n}^{\top} x(T)$ over a finite horizon $T$, under the following constraints:

1) the total MTD is 1 , namely, $\sum_{s=1}^{d} \ell_{s}(t) \leq 1, \forall t$;

2) each drug is either used at its MTD, or not used at all, namely, $\ell_{s}(t) \in\{0,1\}, \forall t, \forall s$.

These constraints imply that a single drug (or a suitable drug combination) is used at a given time at predetermined, clinically relevant doses.

Remark 2: The proposed evolutionary model is a convexmonotone system: it is monotone and its state trajectory is a convex function of both the initial state and the input trajectory. Hence, controller synthesis can be performed by convex optimization, if both the objective function and the constraints are convex [24]. More details can be found in the Appendix.

Typically, plasma drug concentrations require several days to reach a steady state concentration upon treatment initiation. The switching therapy algorithm must therefore select a single treatment at a given time instant and this treatment must also remain constant for intervals of a minimum duration. The drug dose $\ell(t)$ can be rendered piecewise-constant in time over desired intervals by adopting the approach proposed in [19]: for given initial conditions $x(0) \geq 0$ and a given horizon $T$, the time $[0, T]$ is divided into $N$ intervals $T_{1}, T_{2}, \ldots, T_{N}$, and the drug dose choice

$$
\ell(t)= \begin{cases}\ell^{(1)}, & t \in\left[0, T_{1}\right), \\ \ell^{(2)}, & t \in\left[T_{1}, T_{1}+T_{2}\right), \\ \vdots & \\ \ell^{(N)}, & t \in\left[\sum_{i=1}^{N-1} T_{i}, T\right),\end{cases}
$$

is given by the solution of the optimization problem

$$
\begin{aligned}
\min _{\ell^{(1)}, \ldots, \ell^{(N)}} & \mathbf{1}_{n}^{\top} \prod_{i=1}^{N} \exp \left[\left(A-\sum_{s=1}^{d} D_{s} \ell_{s}^{(i)}\right) T_{i}\right] x(0) \\
\text { s.t. } \quad & \ell_{s}^{(i)}(t) \in\{0,1\}, \forall s \in\{1, \ldots, d\}, \forall i \in \mathcal{I}, \\
& \sum_{s=1}^{d} \ell_{s}^{(i)} \leq 1, \quad \forall i \in \mathcal{I}=\{1, \ldots, N\} .
\end{aligned}
$$

A different version of (8), where the constraint $\ell_{s}^{(i)}(t) \in$ $\{0,1\}$ is replaced by $\ell_{s}^{(i)} \geq 0$ (which we will denote as "relaxed (8)"), is relevant for the design of HIV therapies and is a convex optimization problem, as shown in [19]; however, the resulting optimal treatment assumes an additive drug model and requires a combination of drugs at each time interval. This is not a general solution for treatment design with targeted therapies, as discussed earlier, due to the fact that a drug additivity model is not always applicable to any combination of targeted therapies. Undesired side effects due to unmodeled drug interactions could arise. Due to the constraint $\ell_{s}^{(i)}(t) \in\{0,1\}$, convex mixed integer nonlinear programming is required in general to solve (8) (and the more general optimization problem that considers dynamics (5) as well). However, by exploiting the structure of the problem, we can design the optimal switching treatment via convex programming and still enforce the integer constraint.

\section{A. The Diagonal Case: a Convex Programming Approach}

As pointed out in Remark 1, due to the nature of the considered systems, the off-diagonal entries of matrix $A$ are practically negligible with respect to its diagonal entries. If $A$ is a diagonal matrix, the optimal control problem can be solved as a convex optimization program while still making sure that a single drug is used in each interval. In fact, if $A$ is diagonal, the matrices $H_{i}=\left(A-\sum_{s=1}^{d} D_{s} \ell_{s}^{(i)}\right)$ commute and

$$
\mathbf{1}_{n}^{\top} \prod_{i=1}^{N} \exp \left(H_{i} T_{i}\right) x(0)=\mathbf{1}_{n}^{\top} \exp \left(\sum_{i=1}^{N} H_{i} T_{i}\right) x(0) .
$$

Hence, we can write

$$
\mathbf{1}_{n}^{\top} x(T)=\sum_{i=1}^{n} e^{A_{i i} T} \exp \left(-\sum_{s=1}^{d} D_{s} \tau_{s}\right) x_{i}(0),
$$

where

$$
\tau_{s}=\int_{0}^{T} \ell_{s}(t) d t
$$

Since $\ell_{s}(t)$ is either 0 or 1 at each time instant, (11) means that $\ell_{s}(t)$ is 1 for $\tau_{s}$ time units (and 0 otherwise).

The problem can then be equivalently formulated as

$$
\begin{aligned}
\min _{\tau_{1}, \ldots, \tau_{d}} & \sum_{i=1}^{n} e^{A_{i i} T} \exp \left(-\sum_{s=1}^{d} D_{s} \tau_{s}\right) x_{i}(0) \\
\text { s.t. } \quad & \tau_{s} \geq 0, \quad \forall s \in\{1, \ldots, d\}, \\
& \sum_{s=1}^{d} \tau_{s} \leq T,
\end{aligned}
$$


which is a convex optimization problem. The resulting values $\tau_{s}$ are the optimal treatment durations with each of the drugs, $s=1, \ldots, d$, within the given time horizon $T$.

Remark 3: The commutation property (9) means that, when $A$ is diagonal, the minimum final tumor size is achieved regardless of when each drug is used during the treatment horizon, provided that the overall duration of the treatment with drug $s$ is $\tau_{s}$. This additional degree of freedom can be exploited in treatment design.

Since all of the switching rules that use drug $s$ for a total time $\tau_{s}$ lead to the same (minimum) final tumor size, the treatment can be scheduled so as to enforce additional worthwhile properties: e.g., the switchings between different therapy choices can be reduced by applying the drugs sequentially, or the switching law can ensure that the overall tumor size is monotonically decreasing, i.e., $\dot{x}(t)<0 \forall t$.

If $A$ is diagonal, the optimal value of $\mathbf{1}_{n}^{\top} x(T)$ achieved by (12) is the same obtained by solving the relaxed version of (8), as in [19], but it is achieved by using a single drug at a time. When $A$ is not diagonal, we can quantify the cost of allowing only a single therapy to be active at a time. Denote as $C_{\text {rel }}$ the value of $\mathbf{1}_{n}^{\top} x(T)$ obtained by solving the relaxed version of (8), $C_{\text {diag }}$ that obtained by applying the strategy given by (12) (even though $A$ is not diagonal), and $C_{\text {opt }}$ the best value we could achieve by using one drug at a time. Then,

$$
C_{\text {rel }} \leq C_{\text {opt }} \leq C_{\text {diag. }} .
$$

If $A$ is not diagonal, but $C_{\text {rel }}$ and $C_{\text {diag }}$ are close, by adopting the approach in (12) we get close to the "real optimum".

\section{Example: Treatment Strategies for ALK REARranged Non-SMALl CEll Lung CANCER}

Chromosomal translocations of the anaplastic lymphoma kinase (ALK) have been associated in several hematological and solid malignancies including in non small cell lung carcinomas (NSCLC) [30], [31]. The majority of ALK rearrangments described in NSCLC are cases involving fusions of the echinoderm microtubule-associated proteinlike 4 (EML4) gene with ALK [32]. In particular, this abnormal rearrangement leads to the production of a fusion gene (EML4-ALK) that promotes and maintains cancer cell proliferation.

Small molecule inhibitors targeted to the ALK gene have been proposed to address ALK-rearranged NSCLC. While most ALK rearranged lung cancer patients respond initially to treatment with an ALK tyrosine kinase inhibitor (TKI) such as crizotinib, most develop resistance due to the presence of additional genetic alterations, namely secondary mutations on ALK (see Table III) and copy number gain (CNG) in the ALK gene. Other resistance mechanisms include the activation of other signaling pathways such as epidermal growth factor (EGFR), Kirsten rat sarcoma viral oncogene homolog (KRAS) and met proto-oncogene (MET) [33]. Next generation ALK inhibitors such as alectinib, ceritinib and lorlatinib have been proposed to overcome resistance to crizotinib.
Nearly $60 \%$ of ALK-positive NSCLC patients undergoing crizotinib treatment exhibit brain metastasis as the first site of disease progression [34]. Surgery and/or radiation therapy are the primary treatment modalities for most patients with brain metastases from NSCLC, including those with the EML4ALK fusion oncogene [35]. Whole brain radiation therapy (WBRT) is routinely used in patients with multiple brain metastases often exhibits suboptimal local control when used without radiosurgery/surgery in addition to neurocognitive toxicities. Despite the limited drug penetration of early generation of ALK targeted therapies, such as crizotinib, newer generation drugs such as alectinib and lorlatinib have been shown to have increased activity in the central nervous system (CNS).

In this section we demonstrate how the proposed convex optimization based approach allows us to design switching strategies that control tumor progression at best, despite imperfect penetration in different compartments and significant intratumor heterogeneity. We consider four potential inhibitors (crizotinib, alectinib, ceritinib and lorlatinib) as candidates for a switching strategy to potentially control the evolutionary dynamics of nine mutations, listed in Table III that include the EML4-ALK driver oncogene and other mutations have been shown to be resistant to at least one of these four targeted therapies. We assume there are two compartments where disease may be present, the primary tumor site in the lung (compartment 1) and the secondary site in the brain (compartment 2).

Growth and drug effects for every mutation in this study were derived from existing murine tumor models as referenced in Table III. Plasma drug concentrations and CSF to plasma drug concentration ratios are found in Table II. We assume that mutation rates are independent of compartment and migration rates are independent of mutations. Units of concentration in number of cells $/ \mathrm{ml}$ for states and $\mu \mathrm{g} / \mathrm{ml}$ are used for drugs and time is measured in days. The standard volume is $1 \mathrm{ml}$.

We consider several tumor cell initial conditions that correspond to different tumor heterogeneity and progression to secondary tumor sites, representative of varying stages of disease. IC 1 is $x_{0}=10^{7}$. [956 55555555410000000000000$]^{\top}$ (tumor in compartment 1 only, with EML4-ALK-MET and all the other mutations), IC 2 is $x_{0}=10^{7}$.

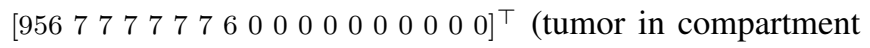
1 only, with all mutations but EML4-ALK-MET), IC 3 is

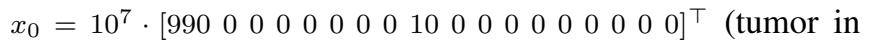
compartment 1 only, with mutant EML4-ALK-MET only),

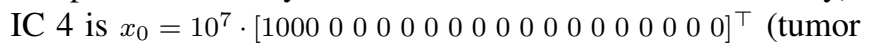
in compartment 1 only, without any mutations), IC 5 is $x_{0}=$

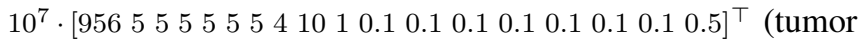
in both compartments, with all mutations), IC 6 is $x_{0}=10^{7}$. [956 7 777777601.50.10.10.10.10.10.10.1 0] ${ }^{\top}$ (tumor in both compartments, with all mutations but EML4-ALK-MET), IC

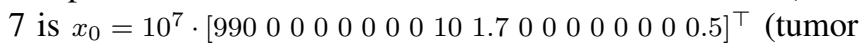
in both compartments, with mutant EML4-ALK-MET only),

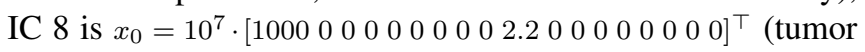
in both compartments, without any mutations). 

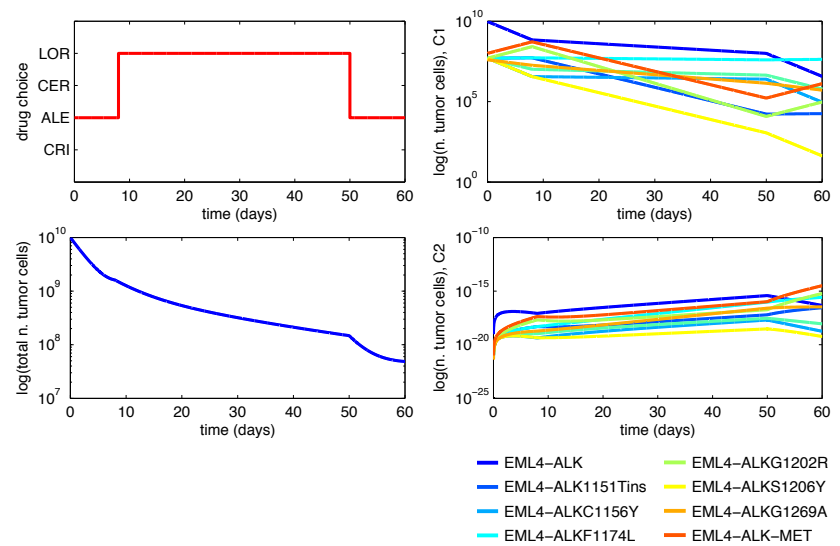

(A)

(B)

Fig. 2. (A) Treatment strategy synthesized by algorithm for a highly heterogeneous tumor in the lung (IC1) and time evolution of the bulk tumor cell population. (B) Evolution of subpopulations in the lung (C1) and brain (C2) for conditions in (A).

Treatment strategies synthesized by the algorithm are shown in Table I. The results obtained with the proposed optimization strategy, in terms of overall tumor reduction, are compared with the results given by the relaxed problem, using a single drug (crizotinib) without switching. We observe that the single drug strategies in our example can not simultaneously control tumor cell migration and heterogeneity but switching strategies can offer better tumor response. In some cases, the overall tumor cell population grows despite switching therapies, due to the presence of subpopulations that are not effectively targeted by any of drugs considered here. However, our switching therapy allows for a much slower increase in tumor size compared to the single constant drug therapy. It is worth pointing out that the performance achieved is almost exactly that achievable by solving the relaxed problem. Hence, in view of the problem structure, namely that $A$ is very close to diagonal, we can obtain the same optimal performance as in the relaxed problem and still enforce the constraint of using one drug at a time. For the initial condition IC 1, Fig. 2 shows a possible therapy scheduling with a 60 days horizon, with drug 2 given for the first 8 days, drug 4 given for the following 42 days and drug 2 given again for 10 days (the overall time per drug is that shown in Table I for this case), along with the corresponding time evolution of the number of tumor cells. Note that the therapy scheduling has been chosen in order to make sure that the overall tumor size is decreasing at any point in time, a desirable property from a clinical perspective. Fig. 2 shows the time evolution of the amount of cancer cells of the different mutant lines in each of the two compartments.

The benefits of switching are well demonstrated by the results of our algorithm. Experimentally searching for timevarying control strategies is infeasible as the number of potential therapies and possible switches to consider is experimentally intractable. We propose to guide these experimental activities with our ability to design and synthesize switching therapy controllers. As such, one could generate optimal controllers tailored to address intratumor heterogeneity in primary and secondary tumor sites, and subsequently verify these predicted strategies in murine models.

\section{CONClusion And Future Work}

In this paper, we introduced an evolutionary cancer model that incorporates replication, mutation and compartmental migration and we showed that a switching therapy that minimizes tumor population can be synthesized by solving a convex optimization problem, exploiting the fact that the evolution matrix is close to diagonal. Importantly, our algorithm specifies the length of time that a drug must be implemented to optimally control tumor progression and this allows for a flexible scheduling strategy that may be more readily implemented clinically. We applied our method to the design of small molecule inhibitor treatment strategies for metastatic ALK-rearranged non small cell lung cancer, compared the outcome obtained with different approaches and showed that switching protocols lead to improvements over standard constant therapies. Future work will focus on several directions: for instance, building more detailed and realistic models of the phenomenon (for instance, allowing mutation rates to depend on the presence of drugs, or including the interplay with the immune system), and optimally scheduling switching treatments, based on the diagonal convex programming formulation, given the total time for which each drug is needed, in order to satisfy additional clinical requirements.

\section{ACKNOWLEDGEMENTS}

This work has been supported by the Swedish Research Council through the LCCC Linnaeus Center and the eLLIIT Excellence Center at Lund University.

\section{REFERENCES}

[1] S. Hoelder, P. A. Clarke, and P. Workman, "Discovery of small molecule cancer drugs: successes, challenges and opportunities," Molecular oncology, vol. 6, no. 2, pp. 155-176, 2012.

[2] R. Nazarian, H. Shi, Q. Wang, X. Kong, R. C. Koya, H. Lee, Z. Chen, M.-K. Lee, N. Attar, H. Sazegar, et al., "Melanomas acquire resistance to b-raf (v600e) inhibition by rtk or n-ras upregulation," Nature, vol. 468, no. 7326, pp. 973-977, 2010.

[3] C. Swanton and R. Govindan, "Clinical implications of genomic discoveries in lung cancer," New England Journal of Medicine, vol. 374, no. 19, pp. 1864-1873, 2016.

[4] R. C. Doebele, A. B. Pilling, D. L. Aisner, T. G. Kutateladze, A. T. Le, A. J. Weickhardt, K. L. Kondo, D. J. Linderman, L. E. Heasley, W. A. Franklin, et al., "Mechanisms of resistance to crizotinib in patients with ALK gene rearranged non-small cell lung cancer," Clinical cancer research, vol. 18, no. 5, pp. 1472-1482, 2012.

[5] J. A. Engelman and P. A. Janne, "Mechanisms of acquired resistance to epidermal growth factor receptor tyrosine kinase inhibitors in nonsmall cell lung cancer," Clinical Cancer Research, vol. 14, no. 10, pp. 2895-2899, 2008.

[6] B. E. Johnson, T. Mazor, C. Hong, M. Barnes, K. Aihara, C. Y. McLean, S. D. Fouse, S. Yamamoto, H. Ueda, K. Tatsuno, et al., "Mutational analysis reveals the origin and therapy-driven evolution of recurrent glioma," Science, vol. 343, no. 6167, pp. 189-193, 2014.

[7] T. S. Mok, Y.-L. Wu, S. Thongprasert, C.-H. Yang, D.-T. Chu, N. Saijo, P. Sunpaweravong, B. Han, B. Margono, Y. Ichinose, et al., "Gefitinib or carboplatin-paclitaxel in pulmonary adenocarcinoma," New England Journal of Medicine, vol. 361, no. 10, pp. 947-957, 2009. 


\begin{tabular}{|c|c|c|c|c|c|c|c|c|}
\cline { 2 - 9 } \multicolumn{1}{c|}{} & \multicolumn{4}{|c|}{ Time horizon $T=60$ days } & \multicolumn{4}{c|}{ Time horizon $T=90$ days } \\
\hline IC & $\tau_{1}, \tau_{2}, \tau_{3}, \tau_{4}$ & $C_{\text {diag }}$ & $C_{\text {rel }}$ & $C_{1}$ & $\tau_{1}, \tau_{2}, \tau_{3}, \tau_{4}$ & $C_{\text {diag }}$ & $C_{\text {rel }}$ & $C_{1}$ \\
\hline 1 & $0,18,0,42$ & $4.869110^{7}$ & $4.868910^{7}$ & $4.788410^{9}$ & $0,12,0,128$ & $2.352510^{7}$ & $2.352510^{7}$ & $1.217110^{12}$ \\
\hline 2 & $0,19,0,41$ & $6.469510^{7}$ & $6.469210^{7}$ & $6.705310^{9}$ & $0,12,0,128$ & $3.273010^{7}$ & $3.271410^{7}$ & $1.704010^{12}$ \\
\hline 3 & $51,0,0,9$ & $6.127010^{3}$ & $6.125910^{3}$ & $7.363710^{3}$ & $90,0,0,50$ & 0.0048 & 0.0048 & 0.0223 \\
\hline 4 & $0,60,0,0$ & 26.8926 & 26.8926 & 63.9979 & $0,140,0,0$ & 0 & 0 & 0 \\
\hline 5 & $0,5,0,55$ & $5.241210^{9}$ & $5.241110^{9}$ & $2.957010^{12}$ & $0,12,0,128$ & $2.809310^{13}$ & $2.809010^{13}$ & $9.492610^{19}$ \\
\hline 6 & $0,14,1,45$ & $1.358910^{9}$ & $1.358910^{9}$ & $5.791210^{11}$ & $0,31,0,109$ & $4.553710^{12}$ & $4.553210^{12}$ & $4.827210^{18}$ \\
\hline 7 & $0,4,0,56$ & $3.375010^{9}$ & $3.375010^{9}$ & $2.463610^{12}$ & $0,1,0,139$ & $5.131110^{12}$ & $5.129210^{12}$ & $9.010310^{19}$ \\
\hline 8 & $0,60,0,0$ & 48.2854 & 48.2854 & $8.381510^{10}$ & $0,140,0,0$ & 0 & 0 & $4.987210^{15}$ \\
\hline
\end{tabular}

TABLE I

Optimal therapy choice $\left(\tau_{i}\right.$ days per drug $\left.i\right)$ and optimal values of $\mathbf{1}_{n}^{\top} x(T)$ obtained with (12) $\left(C_{\text {diag }}\right)$, with the relaxed version of (8) $\left(C_{\text {rel }}\right)$ and with drug crizotinib only $\left(C_{1}\right)$, for eight different initial conditions (IC) and two different time horizons $T$.

\begin{tabular}{|c|c|c|c|c|}
\hline ALK TKI & targeted gene & plasma conc. $\mu \mathrm{M}$ & $\chi$ & ref. \\
\hline crizotinib & MET, ALK & $0.34-0.57^{\circ}$ & 0.0026 & {$[36]$} \\
alectinib & ALK & $0.87-1.21^{\circ}$ & 0.79 & {$[37]$} \\
ceritinib & ALK & $0.62-1.03^{\circ}$ & $0.5^{*}$ & {$[38]$} \\
lorlatinib & ALK & $1.0 \dagger$ & $0.4 \ddagger$ & {$[39]$} \\
\hline
\end{tabular}

TABLE II

Plasma concentrations and cerebrospinal fluid (CSF) to plasma ratios for crizotinib, alectinib, ceritinib and lorlatinib indicate drug concentration differences measured in the blood (and accessible to the primary tumor site) versus the concentration in the CSF, accessible to central nervous system organs (e.g. brain, location of secondary tumor sites). ${ }^{\circ}$ derived from the U.S. FDA approved drugs list [40], *estimate, ceritinib CSF to plasma ratios

have not been studied thusfar, $\dagger$, estimate, ongoing clinical trial, $\dagger$ estimate based on number of patients with partial and complete response and intracranial disease in ongoing clinical trial.

\begin{tabular}{|c|c|c|c|c|c|c|c|c|c|c|}
\hline \multirow{2}{*}{ mutation } & \multirow{2}{*}{$\begin{array}{c}\text { growth } \\
\text { rate day }\end{array}$} & \multicolumn{2}{|c|}{ crizotinib } & \multicolumn{2}{|c|}{ ceritinib } & \multicolumn{2}{|c|}{ alectinib } & \multicolumn{2}{|c|}{ lorlatinib } & \multirow[b]{2}{*}{ ref. } \\
\hline & & ref. & rate day ${ }^{-1}$ & ref. & rate day ${ }^{-1}$ & ref. & rate day ${ }^{-1}$ & ref. & rate day ${ }^{-1}$ & \\
\hline EML4-A & 0.1386 & [41] & 0.45305 & [42] & 0.141885 & [41] & 0.4675 & [43] & 0.18459 & [44] \\
\hline EML4-ALK $^{1151 \text { Tins }}$ & 0.1888 & [43] & 0.142345 & [42] & 0.9563 & [41] & 0.18183 & [43] & 0.3807 & [44] \\
\hline EML4-ALK $^{\mathrm{C} 1156 \mathrm{Y}}$ & 0.1099 & [41] & 0.1144 & [45] & 0.08593 & [41] & 0.4388 & [46] & 0.11868 & [44] \\
\hline EML4-ALK $^{\text {F1174L }}$ & 0.179 & [43] & 0.10706 & [47] & 0.09726 & [41] & 0.17203 & [43] & 0.185948 & [44] \\
\hline EML4-ALK $^{\mathrm{L} 1196 \mathrm{M}}$ & 0.1003 & [44] & 0.1096 & [42] & 0.097015 & [41] & 0.2983 & [43] & 0.1204 & [44] \\
\hline EML4-ALK $^{\mathrm{G} 1202 \mathrm{R}}$ & 0.2067 & [43] & 0.1885 & {$[41] \ddagger$} & 0.14131 & [41] & 0 & [43] & 0.4446 & [44] \\
\hline EML4-ALK $^{\text {S1206Y }}$ & 0.1569 & [43] & 0.3467 & [42] & 0.9244 & [41] & 0.4858 & [43] & 0.3488 & [44] \\
\hline EML4-ALK $^{\mathrm{G} 1269 \mathrm{~A}}$ & 0.198 & [43] & 0.2145 & [41] & 0.6601 & [41] & 0.29702 & [43] & 0.25916 & [44] \\
\hline EML4-ALK-METamp & 0.219 & 1 & 0.37775 & 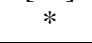 & 0.0123 & 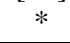 & 0.0123 & 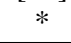 & 0.4109 & \\
\hline
\end{tabular}

TABLE III

ALK mutation growth rates and the effect of ALK inhibitors crizotinib, alectinib, ceritinib and lorlatinib at plasma doses listed in Table II, and derived from existing murine tumor models listed above. †estimated, faster growth of MET amplified cancer cells, *estimate, $\ddagger$ approximation based on IC50.

[8] D. B. Costa, A. T. Shaw, S.-H. I. Ou, B. J. Solomon, G. J. Riely, M.J. Ahn, C. Zhou, S. M. Shreeve, P. Selaru, A. Polli, et al., "Clinical experience with crizotinib in patients with advanced ALK-rearranged non-small-cell lung cancer and brain metastases," Journal of Clinical Oncology, pp. JCO-2014, 2015.

[9] F. Fu, M. A. Nowak, and S. Bonhoeffer, "Spatial heterogeneity in drug concentrations can facilitate the emergence of resistance to cancer therapy," PLoS Comput Biol, vol. 11, no. 3, p. e1004142, 2015.

[10] A. Wu, K. Loutherback, G. Lambert, L. Estévez-Salmerón, T. D. Tlsty, R. H. Austin, and J. C. Sturm, "Cell motility and drug gradients in the emergence of resistance to chemotherapy," Proceedings of the National Academy of Sciences, vol. 110, no. 40, pp. 16 103-16 108, 2013.

[11] L. L. Liu, F. Li, W. Pao, and F. Michor, "Dose-dependent mutation rates determine optimum erlotinib dosing strategies for EGFR mutant non-small cell lung cancer patients," PloS one, vol. 10, no. 11, p. e0141665, 2015.

[12] S.-h. Chen, W. Forrester, and G. Lahav, "Schedule-dependent interaction between anticancer treatments," Science, vol. 351, no. 6278, pp.
1204-1208, 2016.

[13] M. Alamir and S. Chareyron, "State-constrained optimal control applied to cell-cycle-specific cancer chemotherapy," Optimal Control Applications and Methods, vol. 28, no. 3, pp. 175-190, 2007.

[14] S. Chareyron and M. Alamir, "Model-free feedback design for a mixed cancer therapy," Biotechnology progress, vol. 25, no. 3, pp. 690-700, 2009.

[15] M. Alamir, "Robust feedback design for combined therapy of cancer," Optimal Control Applications and Methods, 2012.

[16] B. Zhao, M. T. Hemann, and D. A. Lauffenburger, "Intratumor heterogeneity alters most effective drugs in designed combinations," Proceedings of the National Academy of Sciences, vol. 111, no. 29, pp. 10773-10778, 2014.

[17] B. Zhao, J. R. Pritchard, D. A. Lauffenburger, and M. T. Hemann, "Addressing genetic tumor heterogeneity through computationally predictive combination therapy," Cancer discovery, vol. 4, no. 2, pp. 166-174, 2014.

[18] E. Hernandez-Vargas, P. Colaneri, R. Middleton, and F. Blanchini, 
"Discrete-time control for switched positive systems with application to mitigating viral escape," International Journal of Robust and Nonlinear Control, vol. 21, no. 10, pp. 1093-1111, 2011.

[19] P. Colaneri, R. H. Middleton, Z. Chen, D. Caporale, and F. Blanchini, "Convexity of the cost functional in an optimal control problem for a class of positive switched systems," Automatica, vol. 50, no. 4, pp. 1227-1234, 2014

[20] E. A. Hernandez-Vargas, P. Colaneri, and R. H. Middleton, "Optimal therapy scheduling for a simplified HIV infection model," Automatica, vol. 49, no. 9, pp. 2874-2880, 2013.

[21] _ - "Sub-optimal switching with dwell time constraints for control of viral mutation," in 51st IEEE Conference on Decision and Control (CDC), Dec 2012, pp. 4906-4911.

[22] _ - "Switching strategies to mitigate HIV mutation," IEEE Transactions on Control Systems Technology, vol. 22, no. 4, pp. 1623-1628, July 2014.

[23] F. Blanchini, P. Colaneri, and R. H. Middleton, "A convexity result for the optimal control of a class of positive nonlinear systems," in 19th IFAC World Congress, 2014.

[24] A. Rantzer and B. Bernhardsson, "Control of convex monotone systems," in Decision and Control (CDC), 2014 IEEE 53nd Annual Conference on. IEEE, 2014.

[25] V. Jonsson, A. Rantzer, and R. M. Murray, "A scalable formulation for engineering combination therapies for evolutionary dynamics of disease," in American Control Conference, (ACC), 2014. IEEE, 2014, pp. 2771-2778.

[26] V. Jonsson, N. Matni, and R. M. Murray, "Reverse engineering combination therapies for evolutionary dynamics of disease: An H-inf approach," in Decision and Control (CDC), 2013 IEEE 52nd Annual Conference on. IEEE, 2013, pp. 2060-2065.

[27] M. Eigen, "Viral quasispecies," Scientific American, vol. 269, pp. 4249, 1993.

[28] J. R. Pritchard, P. M. Bruno, L. A. Gilbert, K. L. Capron, D. A. Lauffenburger, and M. T. Hemann, "Defining principles of combination drug mechanisms of action," Proceedings of the National Academy of Sciences, vol. 110, no. 2, pp. E170-E179, 2013.

[29] J. Jia, F. Zhu, X. Ma, Z. W. Cao, Y. X. Li, and Y. Z. Chen, "Mechanisms of drug combinations: interaction and network perspectives," Nature reviews Drug discovery, vol. 8, no. 2, pp. 111-128, 2009.

[30] B. Hallberg and R. H. Palmer, "Mechanistic insight into alk receptor tyrosine kinase in human cancer biology," Nature Reviews Cancer, vol. 13, no. 10, pp. 685-700, 2013.

[31] A. T. Shaw and J. A. Engelman, "Alk in lung cancer: past, present, and future," Journal of Clinical Oncology, vol. 31, no. 8, pp. 1105-1111, 2013.

[32] T. Sasaki, S. J. Rodig, L. R. Chirieac, and P. A. Jänne, "The biology and treatment of eml4-alk non-small cell lung cancer," European journal of cancer, vol. 46, no. 10, pp. 1773-1780, 2010.

[33] H. Isozaki, N. Takigawa, and K. Kiura, "Mechanisms of acquired resistance to ALK inhibitors and the rationale for treating alk-positive lung cancer," Cancers, vol. 7, no. 2, pp. 763-783, 2015.

[34] B. J. Solomon, T. Mok, D.-W. Kim, Y.-L. Wu, K. Nakagawa, T. Mekhail, E. Felip, F. Cappuzzo, J. Paolini, T. Usari, et al., "Firstline crizotinib versus chemotherapy in ALK-positive lung cancer," New England Journal of Medicine, vol. 371, no. 23, pp. 2167-2177, 2014.

[35] D. Maillet, I. Martel-Lafay, D. Arpin, and M. Pérol, "Ineffectiveness of crizotinib on brain metastases in two cases of lung adenocarcinoma with eml4-alk rearrangement," Journal of Thoracic Oncology, vol. 8, no. 4, pp. e30-e31, 2013.

[36] D. B. Costa, S. Kobayashi, S. S. Pandya, W.-L. Yeo, Z. Shen, W. Tan, and K. D. Wilner, "CSF concentration of the anaplastic lymphoma kinase inhibitor crizotinib," Journal of Clinical Oncology, vol. 29, no. 15, pp. e443-e445, 2011.

[37] T. Kodama, M. Hasegawa, K. Takanashi, Y. Sakurai, O. Kondoh, and H. Sakamoto, "Antitumor activity of the selective ALK inhibitor alectinib in models of intracranial metastases," Cancer chemotherapy and pharmacology, vol. 74, no. 5, pp. 1023-1028, 2014.

[38] W. C. Dempke, K. Edvardsen, S. Lu, N. Reinmuth, M. Reck, and A. Inoue, "Brain metastases in nsclc-are tkis changing the treatment strategy?" Anticancer research, vol. 35, no. 11, pp. 5797-5806, 2015.

[39] B. Solomon, T. Bauer, E. Felip, B. Besse, L. James, J. Clancy, et al., "Safety and efficacy of lorlatinib (PF-06463922) from the doseescalation component of a study in patients with advanced ALK+ or ROS1+ non-small cell lung cancer (NSCLC)," in ASCO Meeting Abstracts, vol. 34, 2016, p. 9009.
[40] FDA, U.S. Food and Drug Administration Approved Drug Products, 2016 (accessed September 1, 2016), http://www.accessdata.fda.gov/scripts/cder/drugsatfda/index.cfm.

[41] L. Friboulet, N. Li, R. Katayama, C. C. Lee, J. F. Gainor, A. S. Crystal, P.-Y. Michellys, M. M. Awad, N. Yanagitani, S. Kim, et al., "The ALK inhibitor ceritinib overcomes crizotinib resistance in non-small cell lung cancer," Cancer discovery, vol. 4, no. 6, pp. 662-673, 2014.

[42] R. Katayama, A. T. Shaw, T. M. Khan, M. Mino-Kenudson, B. J. Solomon, B. Halmos, N. A. Jessop, J. C. Wain, A. T. Yeo, C. Benes, et al., "Mechanisms of acquired crizotinib resistance in ALKrearranged lung cancers," Science translational medicine, vol. 4, no. 120, pp. 120ra17-120ra17, 2012.

[43] T. Kodama, T. Tsukaguchi, M. Yoshida, O. Kondoh, and H. Sakamoto, "Selective ALK inhibitor alectinib with potent antitumor activity in models of crizotinib resistance," Cancer letters, vol. 351, no. 2, pp. 215-221, 2014.

[44] H. Y. Zou, L. Friboulet, D. P. Kodack, L. D. Engstrom, Q. Li, M. West, R. W. Tang, H. Wang, K. Tsaparikos, J. Wang, et al., "PF-06463922, an ALK/ROS1 inhibitor, overcomes resistance to first and second generation ALK inhibitors in preclinical models," Cancer Cell, vol. 28, no. 1, pp. 70-81, 2015.

[45] Y. L. Choi, M. Soda, Y. Yamashita, T. Ueno, J. Takashima, T. Nakajima, Y. Yatabe, K. Takeuchi, T. Hamada, H. Haruta, et al., "EML4ALK mutations in lung cancer that confer resistance to ALK inhibitors," New England Journal of Medicine, vol. 363, no. 18, pp. 1734-1739, 2010.

[46] H. Sakamoto, T. Tsukaguchi, S. Hiroshima, T. Kodama, T. Kobayashi, T. A. Fukami, N. Oikawa, T. Tsukuda, N. Ishii, and Y. Aoki, "Ch5424802, a selective ALK inhibitor capable of blocking the resistant gatekeeper mutant," Cancer cell, vol. 19, no. 5, pp. 679-690, 2011.

[47] T. Sasaki, K. Okuda, W. Zheng, J. Butrynski, M. Capelletti, L. Wang, N. S. Gray, K. Wilner, J. G. Christensen, G. Demetri, et al., "The neuroblastoma-associated F1174L ALK mutation causes resistance to an ALK kinase inhibitor in ALK-translocated cancers," Cancer research, vol. 70, no. 24, pp. 10038-10043, 2010.

\section{APPENDIX}

The nonlinear dynamical system

$$
\dot{x}(t)=f(x(t), u(t)), \quad x(0)=a,
$$

where $x(t) \in \mathcal{X} \subset \mathbb{R}^{n}, u(t) \in \mathcal{U} \subset \mathbb{R}^{d}, \mathcal{X}$ and $\mathcal{U}$ are convex sets and (13) has a unique solution $x(t)=\phi_{t}(a, u)$, is monotone if its solution is a monotone function of the initial state $a$ and of the input trajectory $u$, i.e., if

$$
\left(a_{0}, u_{0}\right) \leq\left(a_{1}, u_{1}\right) \Rightarrow \phi_{t}\left(a_{0}, u_{0}\right) \leq \phi_{t}\left(a_{1}, u_{1}\right),
$$

element-wise. If, in addition, every row of $f$ is a convex function, then the system is convex-monotone.

Since matrix $A$ is Metzler, the evolutionary compartment model (5) is a convex-monotone system provided that the elements of $\Psi(\ell)$ are increasing concave functions of $\ell$ (this is clearly true in the linear case; $\psi_{s, i}^{k}\left(\ell_{s}\right)$ as in (2) is not concave in $\ell_{s}$, but it can be rendered concave by considering the new control variable $\left.u_{s}=\left(\ell_{s}\right)^{n_{s}}\right)$. Then, along the lines in [24, Section III], convex-monotonicity of (5) can be shown by means of the logarithmic transformation $z_{j}=\log \left(x_{j}\right)$, where $x_{j}$ denotes a specific state component $x_{i}^{k}$.

For convex-monotone systems, the following result holds.

Theorem 1: [24] If $f$ is a continuously differentiable function and (13) is a convex-monotone system admitting the unique solution $x(t)=\phi_{t}(a, u)$, then each component of $\phi_{t}(a, u)$ is a convex function of $(a, u)$.

Hence, given a convex objective function and convex constraints, control design can be efficiently performed via convex optimization. 